\title{
Walter Demel \\ Adelsstruktur und Adelspolitik in der ersten Phase des Königreichs Bayern
}

\author{
Bayern - eine neugeschaffene Monarcbie obne Adel?
}

Bayern habe „wohl noch zu dem ehemaligen Adel gehörige Familien, aber keinen Adel mehr".

Diese Behauptung stammt nicht etwa vom Jahre 1918, sondern wurde 1810 oder 1811 vom Freiherrn v.Asbeck in einer Sitzung des Geheimen Rats, des höchsten Beratungsgremiums im neugeschaffenen Königreich, aufgestellt ${ }^{1}$. Eine Monarchie ohne Adel? Man denkt möglicherweise an die Anfangsjahre des Napoleonischen Kaiserreiches - aber wann hätte es in Bayern eine Revolution gegeben, die den alten Adel vernichtet hätte? Oder meinte Asbeck vielleicht nur, daß der Adel in diesem neuen Königreich, verglichen mit den Verhältnissen im alten Kurstaat, keinen relativ geschlossenen Stand mehr bildete?

In der Tat hatten ja mediatisierte Fürsten, Reichsritter, adelige Reichsstadtpatrizier, altadelige landsässige Grundherren und neunobilitierte Beamte weder rechtlich noch sozial noch gesinnungsmäßig allzuviel gemeinsam.

\section{Die bayerische Adelsmatrikel: Integration und Purifikation des Adels}

Aber die Integration des neubayerischen Adels war doch schon Ende 1806 begonnen worden, jedenfalls soweit dies rechtlich möglich war ${ }^{2}$, und 1808 hatte dieser Prozeß in der Anlegung einer Adelsmatrikel bereits seinen vorläufigen Abschluß gefunden $^{3}$. Darüber hinaus hatte diese Matrikel auch zur „Purifikation“ des Adels beigetragen, indem sie das Chaos beseitigte, das im 18. Jahrhundert namentlich durch die No-

Alle im folgenden verwendeten Signaturen beziehen sich auf die Bestānde des bayerischen Hauptstaatsarchivs, München. Im einzelnen gilt: $\mathrm{Str}=$ Staatsrat, $\mathrm{MA}=$ Ministerium des Äußeren, MInn $=$ Ministerium des Inneren, $M F=$ Ministerium der Finanzen, HerA $=$ Heroldenamt, $\mathrm{MKu}=$ Kultusministerium, $\mathrm{NM}=$ Nachlaß Montgelas. Weitere Abkürzungen: $\mathrm{VO}=$ Verordnung, Rgbl. = Regierungsblatt, $\mathrm{OE}=$ Organisches Edikt, $\mathrm{H}$. = Heft.

\footnotetext{
${ }^{1}$ Undatierter Geheimratsvortrag Asbecks (wohl Juli/September 1810, spätestens April 1811)(Str 1939).

2 VO v. 31.12.1806 (Rgbl. 1807, Sp. 193-218); Deklaration v. 19.3.1807 (Rgbl. 1807, Sp. $465-490)$.

${ }^{3}$ OE v. 28.7.1808 (Rgbl. 1808, Sp. 2029-2044, hier Sp. 2032f.).
} 
bilitierungstätigkeit der Großen Hofpfalzgrafen und die häufige Adelsusurpation hochrangiger Persönlichkeiten bürgerlicher Herkunft im Adelsrecht eingerissen war. Nun mußten nämlich alle Adelsfamilien unter Nennung ihrer sämtlichen Mitglieder die Berechtigung ihres Titels nachweisen, um nach Zahlung einer gewissen Gebühr diesen Titel mit königlicher Bewilligung behalten zu dürfen. Der Vorstand des Reichsheroldenamts, Ritter v. Lang, schildert uns diesen Vorgang in seinen Memoiren recht drastisch: ,Jeder beinahe, der in Baiern zu einer Civil- oder Militärstelle vorgedrungen war, maßte sich für sich und seine Nachkommen eines adelichen Standes und Namens selbst im höhern Grade /.../ an." Größter Jammer sei nun ertönt „aus den Hütten /!/ derjenigen, welche nicht einmal den untersten Grad ihres angesprochenen Adels mit irgend etwas erweisen konnten, als allenfalls mit Schneiderrechnungen (noch dazu unquittirten), worauf geschrieben stand: Für seine Hochfreiherrliche Gnaden die alten Kleider ausgebessert, wie folgt etc. /... / Auf diesem Wege sind denn an 100 Familien gānzlich zurückgewiesen, andere aus Gnade zugelassen worden, sich ein ausdrückliches königliches Diplom gegen Erlegung der vollen Taxe geben zu lassen“.”

Damit war der bayerische Adel nun zwar sozusagen ein staatlich konzessionierter geworden, aber die rechtlichen Unklarheiten waren beseitigt, eine feste Adelshierarchie war eingerichtet, der „Stand“ als solcher also doch gefestigt. Dies konnte Asbeck demnach nicht gemeint haben.

Was er statt dessen zum Ausdruck bringen wollte, war hingegen offenbar, daß der bayerische Adel als Stand derart an politischem und wirtschaftlichem Einfluß, aber auch an Sozialprestige verloren hatte, daß er als ein Adel ohne wirtschaftliche Macht und ohne eigentliche Privilegien, die allen seinen Mitgliedern gemeinsam gewesen wären, praktisch zum „Nominaladel“ - um diesen Ausdruck Zentners zu gebrauchen" - degradiert worden war.

Die wirtschaftlichen Perspektiven für den bayerischen Adel vor dem Hintergrund der ersten Reformphase (1799-1807)

Seit 1799 waren dem Adel in Bayern innerhalb von nicht einmal zehn Jahren etwa durch die Säkularisation von Reichs- und Ritterstiften und die Auflösung adeliger Korporationen - zahlreiche einflußreiche, prestigeträchtige und z.T. auch einträgliche Positionen verlorengegangen und ebensolche Vorrechte, die für die Versorgung eines Großteils seiner Mitglieder eine Rolle spielten, wie sein Monopol auf bestimmte staatliche und kirchliche Ämter, seine sämtlichen Exspektanzen urd seine Steuerprivilegien. Mag sein, daß diese Opfer, die, wie Montgelas sagte, „commendoit impérieusement l'opinion soutenue par l'exemple presque général d'autres pays "66 großenteils

4 Karl Heinrich v.Lang, Memoiren des Karl Heinrich Ritters von Lang, 2 Bde., (Braunschweig 1841/42) hier II, 174, $176 \mathrm{f}$.

${ }^{5}$ Geheimratsvortrag Zentners vom Mai oder Juni 1811 (Str 1939).

6 Georg Laubmann, Michael Doeberl (Hrsg.), Denkwürdigkeiten des Grafen Maximilian Joseph v. Montgelas über die innere Staatsverwaltung Bayems (1799-1817), (München 1908) 114. 
mehr dem Ziel der Durchsetzung des von einem neuen "Zeitgeist“" geforderten Prinzips der Staatssouveränität ${ }^{7}$ und wenigstens teilweise auch mehr den gebieterischen Ansprüchen des Fiskus gebracht werden mußten, als daß sie ein egalitärer gewordenes gesellschaftliches Bewußtsein erzwungen hätte.

Zusammengenommen jedoch trafen sie jedenfalls einen Stand äußerst hart, der, legen wir die Adelsmatrikel zugrunde, mit ca. 9000-10000 Personen zwar nur rund $0,3 \%$ der bayerischen Gesamtbevölkerung umfaßte ${ }^{8}$, der aber trotz seines damit im Vergleich zum französischen oder gar spanischen Adel viel geringeren Bevölkerungsanteils doch im Durchschnitt wohl kaum wohlhabender als jener war. Schon traditionell waren weder der altbayerische Landadel noch die Reichsritterschaft übermäßig reich. Wenn aber 1815 nur mehr knapp die Hälfte aller bayerischen Adelsgeschlechter über nennenswerten Grundbesitz (z. B. eine Hofmark) verfügte, so bleibt schon in ihrem Fall immer noch 'die Frage offen, ob ihr daraus resultierendes Einkommen für sich allein genommen schon für eine standesgemäße Lebensführung ausreichte, was schätzungsweise nur bei einem Fünftel von ihnen - entsprechend 8-10\% aller Adelsfamilien - angenommen werden darf. Wenn außerdem über $50 \%$ über gar keinen entsprechenden Grundbesitz verfügten, so liegen die Gründe dafür allerdings nur teilweise in dem u.a. durch den rückläufigen Realertrag der Grundrenten und die auch den Adel schwer belastenden Folgen zweier Kriegsjahrzehnte bedingten Zwang zum Verkauf adeliger Güter. Die namentlich während seiner Reichsvikariatsjahre exzessive Nobilitierungspolitik Karl Theodors war nämlich bereits überwiegend (grund-)besitzlosen Mitgliedern seiner „Beamtenschaft“ zugute gekommen.

Nunmehr versiegten plötzlich für diesen ganzen Stand traditionelle Geldquellen, neue finanzielle Belastungen kamen auf ihn zu. Indem die Domkapitel nicht mehr aufgefüllt, viele adelige Stifte säkularisiert und auch für die höheren geistlichen Ämter Laufbahnprinzipien durchgesetzt wurden ${ }^{9}$, verlor der geistliche Dienst für den Adel stark an Attraktivität ${ }^{10}$, nahm aber auch der Druck speziell der Kadetten, also der

${ }^{7}$ Dazu Ludwig Doeberl, Maximilian von Montgelas und das Prinzip der Staatssouverānitāt, (München 1925).

B 1818 besaß Bayern - ohne die Pfalz - nach den Angaben von Wolfgang Köllmann (Hrsg.), Quellen zur Bevölkerungs-, Sozial- und Wirtschaftsstatistik Deutschlands 1815-1875, Bd. 1: Antje Kraus (Bearb.), Quellen zur Bevölkerungsstatistik Deutschlands 1815-1875, (Forschungen zur deutschen Sozialgeschichte II/1, Boppard/Rh. 1980) 76, 3261798 Einwohner. Der von mir angegebenen $Z$ ahl bayerischer Adeliger liegen ebenso wie bei den weiter unten ( $S$. 226f.) gemachten Angaben Zählungen bzw. Schätzungen vor, die anhand der bayerischen Adelsmatrikel im bayerischen Hauptstaatsarchiv von mir vorgenommen wurden. Detailliertere Nachweise werde ich demnächst in meiner Arbeit „Gesellschaftsstruktur und Gesellschaftspolitik in der ersten Phase des Königreichs Bayern" (Arbeitstitel) erbringen. Der vorliegende Beitrag stellt nur ein vorweggenommenes und daher auch sehr vorläufiges Resümee eines Teils dieser Arbeit dar.

${ }^{9}$ Geistliche wurden ja "nicht als bloße Kirchendiener, sondern zugleich als Staatsbeamte“ betrachtet. VO v. 7.5 .1804 (Rgbl. 1804, Sp. 510).

${ }_{10}$ Unter den Studierenden am wichtigsten staatlichen Priesterseminar, dem Landshuter Gregorianum, befanden sich wāhrend der Ära Montgelas offenbar kaum mehr Adelige. Vgl. z. B. Rgbl. 1806, S. 325 f.; Rgbl. 1810, Sp. 1355 f. Entsprechend geringen Zulauf hatte die theologische ganz im Gegensatz zur juristischen - Fakultăt der Universität Landshut von seiten der Söhne aus dem ${ }_{n g}$ efreyten oder siegelmäßigen Stande“. Vgl. Rgbl. 1804, Sp. 305-308; 1806, S. 120; 1807, Sp. 835f.; MInn 23874/I. 
nachgeborenen Söhne, und der Töchter auf ihre Väter zu, ihnen doch anderweitig einen gesicherten Lebensunterhalt zu gewährleisten, z. B. durch Auswerfung eines entsprechenden Heiratsguts oder durch eine mit dem Erstgeborenen gleichberechtigte Teilhabe am Erbe. Gleichzeitig mußte auch nur eine Annäherung der Steuerbelastung adeliger Güter an das allgemeine Niveau nicht nur das Nettoeinkommen der noch grundbesitzenden Adelsfamilien merklich reduzieren ${ }^{11}$, sondern infolge des nunmehr sinkenden Verkaufswerts adeliger Güter und Rechte auch deren Vermögen. Der heftige Widerstand führender altbayerischer Grundherren gegen ihre ab 1807 im Zuge einer größeren Reform eingeleitete steuerliche Gleichstellung ist demnach nur zu verständlich $^{12}$. Sehen wir von einer gewissen Sonderbehandlung der Mediatisierten, mit denen Bayern ohnehin bekanntlich vergleichsweise sehr milde verfuhr, ab, so blieben diese Beschwerden äußerlich gesehen erfolglos. Ab 1814 zahlten selbst die Dominikalisten exakt die gleiche Steuerquote wie sämtliche Rustikalbesitzer. Doch da gleichzeitig die Kapitalanschläge der Dominikalrenten erheblich gesenkt wurden, bleibt die Frage offen, ob hier wirklich, wie es im Text der Verordnung heißt, „das billige Verhältnis zwischen der Grund- und Dominikalsteuer nicht verrückt" wurde ${ }^{13}$.

Denn das praktische Ergebnis der damals angeordneten Modifikationen scheint in einer unverhältnismäßig hohen Senkung der Dominikal- gegenüber der Grundsteuer bestanden zu haben ${ }^{14}$. Hatten die Reklamationen, die auch zuvor schon recht entgegenkommend behandelt worden zu sein scheinen ${ }^{15}$, also vielleicht doch letztendlich den Erfolg, das Prinzip der Gleichbesteuerung etwas ins Wanken gebracht zu haben? In jedem Fall ist festzustellen, daß die Einbeziehung des Adels in eine neue, zweifellos gleichheitlichere Steuergesetzgebung keineswegs unvermittelt, sondern nur ganz behutsam, d. h. schrittweise, erfolgte.

Ebensowenig läßt sich beispielsweise im Zusammenhang mit der Säkularisation, wo etwa für Domherren beträchtliche Pensionen ausgesetzt wurden, während Klosterhandwerker für den Verlust ihres Arbeitsplatzes normalerweise bei weitem keine ausreichende Entschädigung erhielten ${ }^{16}$, behaupten, die Regierung wäre bei ihren ein-

${ }^{11}$ Anonym/Jobann Nepomuk Frbr. v. Pelkoven, Ueber die Anwendung des Gleichheits-Prinzips bey dem Steueranschlage der Ritter- und Bauerngüter, ein Nachtrag zu den Aktenstücken über das momentane Steuerprovisorium in der Provinz Baiern, (o.O. 1808) bes. $12 \mathrm{ff}$.; Anonym/Ders., Ueber Fassionen und direkte Auflagen. Von einem baierischen Edelmann, (o. O. 1808) bes. $29 \mathrm{ff}$. Andererseits gab es offenbar für den Adel kaum mehr die Möglichkeit, seine Einnahmen zu steigern. Dazu für Franken: Hanns Hubert Hofmann, Adelige Herrschaft und souveräner Staat. Studien über Staat und Gesellschaft in Franken und Bayern im 18. und 19. Jahrhundert, (Studien zur bayerischen Verfassungs- und Sozialgeschichte 2, München 1962) 134.

12 MF 10045; MF 16685.

${ }^{13}$ VO v. 18. 2.1814 (Rgbl. 1814, Sp. 345-350, zit. Sp. 346).

14 Dies geht aus den Vergleichstabellen MF 10191 klar hervor.

${ }^{15}$ Edikt v. 30. 9.1811 (Rgbl. 1811, Sp. 1521-1550), geändert durch VO v. 15.1.1813 (Rgbl. 1813, Sp. 513-518), diese allerdings wiederum modifiziert durch VO v. 9.1.1814 (Rgbl. 1814, Sp. 81-83).

${ }^{16}$ Anton Scbneider, Der Gewinn des bayerischen Staates von säkularisierten landständischen Klöstern in Altbayern, (Miscellanea Bavarica Monacensia 23, München 1970) $89 \mathrm{ff}$. Hingegen wurden beispielsweise auch die ehemaligen altbayerischen Landschaftsverordneten durchaus großzügig abgefunden. Geheime Staatskonferenz v. 22.9.1808 (Str 8). 
deutig hier mehr staats- als gesellschaftspolitisch motivierten Maßnahmen gerade gegen die betroffenen Adeligen besonders rücksichtslos vorgegangen. Montgelas hat derartige Tatsachen seinen adeligen Kritikern denn auch immer wieder vorgehalten.

\section{8 - Ein neuer Adel für ein neues Königreich?}

Trotzdem scheint selbst im heutigen Rückblick das Jahr 1808 eine Wende der bayerischen Adelspolitik gebracht zu haben. Die Errichtung einer Adelsmatrikel, die Betonung des Willens zur Gleichbesteuerung, ja selbst die Beseitigung diverser Feudalrechte eindeutig herrschaftlichen, d.h. personalen Charakters - wie der persönlichen Leibeigenschaft oder des Gesindezwangs - konnte, aber mußte man noch nicht als spezifisch ,adelsfeindlich“ verstehen. Schieres Entsetzen mußte es jedoch in weiten Kreisen des bayerischen Adels auslösen, daß die Regierung gleichzeitig auch noch die Einführung eines modifizierten Code Napoléon im Königreich vorbereitete, ankündigte, daß mit dem Inkrafttreten dieses Gesetzbuches das Institut der Siegelmäßigkeit erlöschen sollte, daß sie dem Adel jede streitige Gerichtsbarkeit entzog und die Aufhebung der Fideikommisse sowie eine Konstitution verkündete, von der Asbeck später sagte, daß bei ihrem Entwurf die Staatsführung unmöglich von der Absicht geleitet gewesen sein könnte, „noch ferner einen Adel haben zu wollen“17.

Doch Asbeck befand sich im Irrtum. Am Tage der Verabschiedung des Adelsedikts von 1808, durch das ein neues Adelsrecht ins Leben trat, erklärte Montgelas selbst mit dem Ausdruck persönlichen Bedauerns, „daß er ohngeachtet seines reifesten Nachdenkens keinen rechten Ausweg habe finden können, diesen Gegenstand den dermaligen Verhältnißen anpaßend und für die bestehende /sic/ Adeligen nicht zu hart zu behandeln"18. Warum kam es dann überhaupt zu so plötzlichen, weitreichenden Veränderungen im bayerischen Adelsrecht? Die Antwort lautet: wegen des Drucks von seiten Napoleons.

Denn nach der Mailänder Konferenz glaubte man in München, die bayerischen Verhältnisse so weit als möglich den französischen angleichen zu müssen. Dabei schätzte Montgelas offenbar von Anfang an den politischen Spielraum für Abweichungen vom französischen Vorbild noch als größer ein als die meisten seiner Mitarbeiter, und er war auch eher zum Widerstand gegen den Kaiser bereit. So haben er und seine Ministerkollegen damals manche noch radikalere, am nachrevolutionären französischen Rechtszustand orientierte Vorschläge ihrer großenteils bürgerlichen Geheimen Referendäre abgeblockt, und auf Veranlassung Justizminister Morawitzkys hin entstand sogar - was bisher unbekannt geblieben ist - auch in Bayern ein Gesetzentwurf, durch den das Institut der Emphyteuse in reformierter Form in den modifizierten Code Napoléon hätte eingebaut werden sollen ${ }^{19}$.

Montgelas erkannte nämlich wohl klarer als andere den Widerspruch zwischen $\mathrm{Na}$ poleons eigener neuer Adels- und Majoratspolitik und seinen anderweitigen Bestre-

${ }_{17}$ Vortrag Asbecks, (wie Anm. 1); vgl. Wilbelm Volkert, Marginalien zur bayerischen Geschichte 1800-1810, in: ZBLG 25 (1962) 414-469, bes. 423.

${ }^{18}$ Geheime Staatskonferenz v. 28.7.1808 (Str 8). 
bungen, auch in Deutschland die überkommenen Agrarstrukturen zu beseitigen ${ }^{20}$, er konnte sich darüber hinaus schon frühzeitig, nämlich bereits in Mailand, wie auch später in Erfurt, persönlich davon überzeugen, daß Napoleon an einem Ruin zahlreicher bayerischer Adelsfamilien nichts gelegen war ${ }^{21}$, und er erkannte schließlich schon früher als die meisten seiner Zeitgenossen, daß der Stern des Kaisers mit dem Beginn seines Spanienabenteuers zu sinken begann. Dies bedeutete nun aber zwar einerseits, daß sich der Handlungsspielraum der Münchner Regierung gegenüber Napoleon wieder vergrößerte, jedoch andererseits, daß künftig auch in der Innenpolitik eher Rücksicht zu nehmen sein würde auf einen eventuellen Partner Österreich ${ }^{22}$ und ebenso auf eine in altständischen Kategorien denkende „Opposition“ im Geheimen Rat, bestehend aus den Grafen Arco, Törring und Preysing. Wie heftig diese Gruppe über die legislativen Entwürfe eines Feuerbach herzog, beweist, daß die von H. H. Hofmann überspitzt als „latenter Klassenkampf zwischen Feudalismus und Bürokratie“ bezeichnete Rivalitāt zwischen dem im Staatsdienst aufgestiegenen Bildungsbürgertum und einem teilweise noch sehr standesbewußten grundherrlichen Adel in Bayern bis in die Reihen des Geheimen Rats reichte.

Montgelas jedoch, der 1808 dafür gekämpft hatte, daß sich der Übergang vom Alten zum Neuen für den bestehenden Adel nicht zu gravierend auswirkte, kam in der Folgezeit den Wünschen etwa eines Grafen Törring nur sehr bedingt nach. Denn die wirtschaftliche Lage des Adels erforderte nach seiner Ansicht nun mehr denn je eine durchgreifende Adelsreform, und nach den einmal notwendigen, nur gewissermaßen zu plötzlich erfolgten schwerwiegenden rechtlichen Eingriffen in dessen Verhältnisse schien ihm nun dazu der Weg frei. Die Idee einer Umstrukturierung des bayerischen Adels nach englischem Vorbild klingt in verschiedenen Äußerungen damaliger Politiker $a^{23}$, und man kann es durchaus als ersten Ansatz in dieser Richtung betrachten, wenn am 19. Mai 1808 fast 40 Personen - meist hohe Beamte wie Feuerbach oder Gönner, aber auch Gelehrte (wie Jacobi) - in den neugeschaffenen Zivilverdienstorden der Krone aufgenommen wurden ${ }^{24}$ und damit einen „Ritter"-Stand erwarben, den sie nach dessen 1812 erfolgter Gleichstellung mit dem alten Ritterstand, der vierthöch-

19 Dazu Walter Demel, Der bayerische Staatsabsolutismus 1806/08-1817. Staats- und gesellschaftspolitische Motivationen und Hintergründe der Reformāra in der ersten Phase des Königreichs Bayern, (München 1983, 40 ff., $479 \mathrm{ff}$. Der Entwurf findet sich unter der Signatur MF 602.

${ }^{20}$ Helmut Berding, Napoleonische Herrschafts- und Gesellschaftspolitik im Königreich Westfalen 1807-1813, (Kritische Studien zur Geschichtswissenschaft 7, Göttingen 1973) 15: „Die Zerstörung der überkommenen feudalen, guts- oder grundherrlichen Agrarstrukturen also, der eigentliche Kern der sozialen Umwandlung während der Französischen Revolution, widersprach einem zentralen gesellschaftlichen Interesse des Kaisers: dem Aufbau einer auf agrarischem Grundbesitz berruhenden imperialen Führungsschicht.“

${ }^{21}$ Undatierte Denkschrift Montgelas' an den König, /Jan. 1808/ (MA 12); Ludwig Graf v. Montgelas, Maximilian Frbr. v. Freyberg-Eisenberg (Hrsg.), Denkwürdigkeiten des bayerischen Staatsministers Maximilian Grafen von Montgelas 1799-1817, (Stuttgart 1887) 171.

${ }^{22}$ Diesen Hinweis verdanke ich meinem verehrten Lehrer, Herrn Prof. Dr. E. Weis.

23 Vgl. z.B. v. Lang, (wie Anm. 4) 186, oder die Äußerungen von Montgelas' Schwager C. Graf Arco bei den Verfassungsberatungen 1814/15: Protokoll v. 15.12.1814 (Str 1645).

24 Vgl. Rgbl. 1808, Sp. 1039-1046; MA Ordensakten 348, 354a, 355. Die Zahl der Gesamtauf- 
sten der ursprünglich sechs Adelsklassen umfassenden bayerischen Adelshierarchie, nur auf einen einzigen ihrer Söhne - und dies noch dazu nur mit königlicher Bewilligung - vererben durften ${ }^{25}$. Das Datum dieser Ordensgründung weist freilich mehr noch in eine andere Richtung: auf die am 1.3.1808 von Napoleon ins Leben gerufene Noblesse impériale ${ }^{26}$.

Gerade um diesem Neuadel eine wirtschaftliche Basis zu schaffen, hatte Napoleon sich aber für seine umfangreiche, größtenteils auf Kosten ausländischer Domänen durchgeführte Dotationspolitik im Majoratsrecht ein neues Rechtsinstitut geschaffen, das nun, nach der Übertragung zahlreicher für den bayerischen Adel destruktiver Elemente des französischen Rechtszustandes auf Bayern, dessen Regierung ebenfalls ein Instrumentarium bot, ihrem Adel - wenigstens einem Teil des neuen und des alten auf der Grundlage von einigen Dotationen, mehrheitlich jedoch von bestehenden Fideikommissen auch für die Zukunft Einfluß und Ansehen zu erhalten bzw. neu zu verschaffen.

\section{Das bayerische Majoratsrecht}

Den Kernpunkt seiner Motive zur Einleitung einer Adelsreform formulierte Montgelas einmal folgendermaßen: es sei nicht seine Absicht, dem Adel „an seinen persönlichen Vorzügen, in so weit sie der Eigenschaft als Staatsbürger nicht widersprechen, etwas nehmen zu wollen, wohl aber den Adel zu souteniren und ihn auf jene zu beschränken, die im Stande sind, ihn mit Würde zu führen, denn er kenne nichts Traurigeres als einen armen Edelmann"27.

Konkret bedeutete dies: unter teilweiser personeller Umstrukturierung sollte der bayerische Adel wenigstens in seiner Spitze ein privilegierter Stand werden, der zwar keine „öffentlichen“ Rechte sui juris mehr ausübte, der aber dank seines politischen und wirtschaftlichen Einflusses und seines allgemeinen Ansehens seine staatstragende Funktion besser als bisher erfüllen konnte. Wie ein Teil seiner Mitarbeiter glaubte der Minister dabei an die Notwendigkeit einer Verbindung von Vermögen einerseits speziell Grundvermögen, da dieses als am stabilsten galt - und adeligen Vorrechten (Titulatur, Zugang zum Hof etc.) andererseits. Denn er spürte offenbar die Tendenz, daß in der liberalen Leistungsgesellschaft des 19. Jahrhunderts der ökonomische Aspekt für das soziale Ansehen einer Person eine zunehmend wichtigere Rolle spielen würde - und zwar auf Kosten rechitlicher Kriterien, wie z. B. einer nachweisbar langen Reihe adeliger Vorfahren ${ }^{28}$.

nahmen an diesem Tag betrug 94, davon waren jedoch 55 bereits adelig. Weitere Ernennungen z. B. Rgbl. 1813, Sp. 683-692; 1815, Sp. 107-112.

${ }^{25}$ VO v. 23.12.1812 (Rgbl. 1813, Sp. 5-9); Entwurf HerA 127.

${ }^{26}$ Pierre Durye, Les chevaliers dans la noblesse impériale, in: La France dans l'époque napoléonienne, (Revue d'histoire moderne et contemporaine 17, 1970) 671-679; Jacques Godechot, Les institutions de la France sous la révolution et l'empire, (Paris ${ }^{2}$ 1968) $696 \mathrm{ff}$.

${ }^{27}$ Protokoll der Geheimratssitzung v. 6.9.1810 (Str 191).

281808 meinte zum Beispiel der Geheime Rat Anselm (Ritter v.) Feuerbach, bekannt als Schöpfer des bayerischen Strafgesetzbuchs von 1813, es sei ,der spanische Hidalgo mit seinem Degen 
$\mathrm{Da}$ in diesem Punkt mehr traditionell denkende Politiker gegen diese im Grunde sehr weitsichtigen Vorstellungen Montgelas' erhebliche Vorbehalte hatten, zeigen die über zwei Jahre andauernden Auseinandersetzungen um die Ausgestaltung des Majoratsrechts ${ }^{29}$, bei denen sich zwei Grundrichtungen gegenüberstanden:

Den einen sollten Majorate primär dazu dienen, möglichst vielen Adelsfamilien eine bestimmte, d.h. in der Regel ihre jetzt (noch) vorhandene wirtschaftliche Basis über Generationen hinweg zu garantieren und dadurch den bereits vorhandenen Erbadel so weit als möglich zu erhalten. Dann aber mußte man relativ zahlreiche Güter dem freien Verkehr entziehen, konnte den Töchtern und Kadetten praktisch keine neuen Versorgungschancen aufgrund eines ausgedehnteren Erbanspruchs eröffnen und konnte auch nicht den Fideikommißbesitzern weitergehende politische Vorrechte, wie z.B. das Recht auf einen Sitz in den geplanten Repräsentativkörperschaften, einräumen. Praktisch hätten - vom Übergang vom privat- zum öffentlich-rechtlichen Charakter und der neugeschaffenen relativen Freiheit des Majoratskonstituenten bei der Auswahl seines Nachfolgers abgesehen - die Fideikommisse beim Úbergang in Majorate nur ihren Namen, nicht ihre Funktion verändert. Diese Position - man könnte sie die „altstāndische“ nennen - vertrat etwa Graf Reigersberg, seit 1810 Justizminister ${ }^{30}$.

Montgelas dagegen wollte eine Adelsreform, nämlich im Sinne einer Zweiteilung des Adels. Die wirtschaftlich potenten Familien sollten in gewisser Angleichung an die Rechtsstellung der Standesherren als Majoratsadel seine Spitze bilden, privilegiert, angesehen, politisch einflußreich - eine Stütze für den monarchischen Staat. Die zweite Adelsklasse, der arme bzw. verarmte Adel, sollte demgegenüber nur eine „Pflanzschule ${ }^{\text {" } 31}$ des ersten darstellen, indem man ihn über seine verbliebenen Mittel frei disponieren ließ und so dem einzelnen Adeligen die Möglichkeit gab, seine wirtschaftlichfinanzielle Regeneration direkt in einen politisch-rechtlichen Aufstieg umzusetzen.

Ein Mittelweg zwischen beiden Positionen, nämlich die Errichtung verschiedener Majoratsklassen mit unterschiedlichen Vorrechten, erwies sich nicht zuletzt deshalb als nicht gangbar, weil die Regierung hier eine neue Adelshierarchie hătte schaffen müssen, die ihrer bisherigen Einteilung in Fürsten, Grafen etc. widersprochen hätte.

So drehte sich die ganze verschlungene Diskussion um die Neugestaltung des Majoratswesens letztlich um die Frage, wie groß der Kreis der Privilegierten sein sollte und wieviele Privilegien man dann dementsprechend diesem Kreis zumessen konnte. Nur $z$ wei Kernfragen seien hier angesprochen: 1. Sollten alte Fideikommisse einer be-

hinter seinem Pflug nicht mehr als - ein Bauer“. Paul Jobann Anselm Feuerbacb, Betrachtungen über den Geist des Code Napoléon und dessen Verhältniß zur Gesezgebung und Verfassung teutscher Staaten überhaupt und Baierns insbesondere, in: Ders. (Hrsg.), Themis oder Beiträge zur Gesezgebung, (Landshut 1812) 3-73, zit. 35. Dieser 1812 veröffentlichte Beitrag war identisch mit einem 1808 erstellten Gutachten, mit Hilfe dessen Feuerbach damals Montgelas seine Vorstellungen von einer neuen bayerischen Zivilgesetzgebung auf der Grundlage des Code Napoléon dargelegt hatte.

${ }^{29}$ Dazu z. B. Str 191, 192, 193, 1939.

${ }^{30} \mathrm{Vgl}$. Votum Reigersbergs v. 24.7.1811 (Str 232).

${ }^{31}$ Diesen Ausdruck gebrauchte der Geheime Rat v. Aretin, Beilage II zum Protokoll der Geheimratssitzung v. 22.5.1811 (Str 1939). 
stimmten Mindestgröße direkt in Majorate übergeführt werden können, oder sollten alle Fideikommisse mit einem Schlag allodifiziert werden, um erst nach der Tilgung der Schulden ihrer Besitzer und der Sicherstellung des selbst bei vielleicht vermindertem Pflichtteil nunmehr sprunghaft gewachsenen Erbanspruchs von seiten der Töchter und der Kadetten den Rechtscharakter von Majoraten zu erlangen, falls dann dazu noch genügend Grundbesitz vorhanden sein sollte? 2. Wie hoch war die „Normalrente" für eine Majoratsbildung anzusetzen, also jene Nettoertragssumme, die der gebundene Besitzkomplex im Jahr mindestens abwerfen sollte? Diese Summe setzte letztlich die Grenze fest zwischen Majoratsadel und übrigem Adel. Obwohl Montgelas im Geheimen Rat erhebliche Widersprüche von seiten einer in den Bahnen adeliger Solidarität denkenden Opposition erleben mußte und auch der König schon aus Mitgefühl nicht wünschte, einen zu großen Teil seines alten Adels entrechtet und ihn den Stürmen eines liberalisierten Wettbewerbs ausgesetzt zu sehen, folgte das Majoratsedikt von $1811^{32}$ bei allen Kompromissen doch im Kern mehr der Linienführung Montgelas'. Zwar betrachtete er mit anderen zusammen den Majoratsadel immer noch als zu wenig exklusiv, aber dieser schien immerhin doch elitär und wohlhabend genug, daß die Regierung nun $i b m$ bestimmte Gerichtsrechte, die sie $1808 \mathrm{dem}$ Adel allgemein entzogen hatte, wieder verleihen konnte.

\section{Die gutsherrliche Gerichtsbarkeit in Bayern}

Damals war nämlich im Zuge der endgültigen Enteignung der eigenberechtigten Gewalten die Patrimonialgerichtsbarkeit sozusagen mediatisiert, also in ihrem Charakter als ius delegatum fixiert, und im übrigen (mit Ausnahme der Standesherrschaften) auf gewisse Polizeibefugnisse und nichtstreitige bürgerliche Rechtsfälle beschränkt worden. Wenn nun gerade dieser zweifellos einträgliche Teil der Zivilgerichtsbarkeit den sog. „Gutsherren“ erhalten blieb, so deshalb, weil die Minister zu dieser Zeit den für die gesetzmäßige Durchführung einer streitigen Gerichtsbarkeit notwendigen Aufwand an Gerichtspersonal etc. als für die meisten der vorhandenen Patrimonialgerichtsherren finanziell zu belastend beurteilten. Die gutsherrliche Gerichtsbarkeit in der Form von 1808 war demnach im Grunde nichts anderes als ein staatlicher Beitrag zur finanziellen Sanierung adeliger Gerichtsherrschaften.

Um den ab 1812 sich herausbildenden Majoratsadel nun aber auch entsprechend mit Einfluß und Ansehen auszustatten, lag es nahe, seine Gerichtsrechte in etwa auf die Stufe derjenigen der Standesherren zu erheben, wobei Napoleon selbst die Bresche in den bisherigen Rechtszustand schlug, indem er den Grafen Wrede in dem bald darauf an Bayem abgetretenen Land Salzburg ein Majorat als kaiserlich-französisches Lehen verlieh, das nicht nur mit der streitigen Zivil-, sondern selbst mit der Strafgerichtsbarkeit ausgestattet war $^{33}$. Die vor allem rechtlich motivierten Bedenken aus

32 OE v. 22.12.1811 (Rgbl. 1812, Sp. 5-54). Die Präambel dieses Edikts gibt die gesetzgeberischen Motive durchaus offen und zutreffend wieder.

${ }_{33}$ Anerkennung durch die kgl. Erklärung v. 5.8.1811 (Rgbl. 1811, Sp. 977-993). Zur Bedeutung dieses Vorgangs vgl. Vortrag Zentners v. Jan. 1811 (Str 8216) und das anonyme, undatierte Ministerialgutachten in MInn 43905. 
dem Justizministerium ${ }^{34}$, derartige Souverānitätsrechte auf der Basis ablösbaren Grundeigentums an Privatpersonen zu verleihen, schob Montgelas nicht zuletzt aus fiskalischen Gründen beiseite 35 .

Ein Edikt vom Jahr $1812^{36}$ schuf demnach

1. „Herrschaftsgerichte 1.Klasse“, speziell, aber nicht ausschließlich für die Mediatisierten, denen ja aufgrund früherer Zusagen sogar die erste Instanz in Straf- und Konsistorialgegenständen zustand,

2. gleichberechtigt mit den Landgerichten „Herrschaftsgerichte 2. Klasse“ für die Majoratsherren und die ihnen gleichgestellten Inhaber größerer Kanzleilehen, wie diejenigen 1. Klasse mit mindestens 300 gerichtsuntertänigen Familien, und

3. die weiterhin den Landgerichten untergebenen und auf die nichtstreitige Gerichtsbarkeit und gewisse Polizeirechte beschränkten „Ortsgerichte“ mit mindestens 50 Familien.

Daß die beim Einbau der Herrschaftsgerichte in die staatliche Gerichtsorganisation zur Purifikation der Gerichtsbezirke notwendigen Maßnahmen wie Infeudation und Austausch von unmittelbar-staatlichen Gerichtsholden zu einer regelrechten Spekulation mit Gerichtssassen führten, zwang die Regierung freilich bald zu Gegenmaßnahmen $^{37}$, die aber nichts daran änderten, daß im Jahre 1817 nicht weniger als $15,8 \%$ der im rechtsrheinischen Bayern lebenden Familien einem Patrimonialgerichtsherrn ${ }^{38}$ unterstanden. Schon im Zusammenhang mit den Verfassungsplänen von 1814/15 hatte Montgelas darauf gedrängt, aus Angehörigen der schon früher als "geborene“ Mitglieder künftiger Repräsentationskörperschaften bezeichneten Führungsschicht des bayerischen Adels - Standesherren und Majoratsbesitzer - eine erste und unter Einbeziehung der übrigen Inhaber von Herrschafts- und Ortsgerichten eine zweite Kammer zu schaffen $^{39}$ - ein für den bayerischen Konstitutionalismus richtungsweisender Gedanke, der ohne die Betrachtung der vorangegangenen Entwicklung im bayerischen Adelsrecht nicht voll verständlich wird: der wohlhabende Adel sollte auch politisch privilegiert werden.

\footnotetext{
${ }^{34}$ Votum Reigersbergs v. 24.7.1811 (Str 232).

${ }^{35}$ Protokoll der Geheimratssitzung v. 4.10.1810 (Str 195). Montgelas äußerte hier gegen die immer wieder vorgetragenen Bedenken gegen die sogar noch über die Weiterexistenz des gerichtsherrlichen Rechts auf Selbstexekution grundherrlicher Gefälle hinausgehende enge Verbindung von Grund- und Gerichtsherrschaft, er "überzeuge sich immer mehr" von der Unschädlichkeit, ja sogar der finanziellen Nützlichkeit einer reformierten Patrimonialgerichtsbarkeit für den Staat. Anlaß dieser Äußerung war übrigens eine Rekurssache des Geheimen Rats Graf Törring gegen die Einschränkung seiner Gerichtsrechte. Vgl. Vortrag C. Graf v. Arcos v. 26.9.1810 (Str 8234).

${ }^{36}$ OE v. 16.8.1812 (Rgbl. 1812, Sp. 1505-1556), dazu Gesetzesmaterialien Str 276-278.

${ }^{37} \mathrm{Vgl}$. Zusammenfassung der Jahresberichte der Innenministerialsektionen für 1816/17 und 1817/18 (MInn 44704), dazu z. B. MInn 30128.

${ }^{38}$ Nach einer Ubersicht v. 15.9.1817 (MF 19712).

${ }^{39}$ Instruktion v. 17.9.1814 (MA 70311), dazu Konzept Montgelas': NM 149. Vgl. die Stellungnahme Sutners (mit Beilage): Str 1642. Schon die Deklaration v. 19.3.1807 (Rgbl. 1807, Sp. 465-490, hier Sp. 467) wollte die Mediatisierten als einen Teil der „ersten Klasse des Adels“ ansehen, bereits 1808 aber beschloß man, in ein Edikt bei der Erwāhnung der Mediatisierten den Zusatz anzufügen: „/.../ und welchen Wir Ihnen etwa gleich zu stellen für gut befinden werden.“ Geheime Staatskonferenz v. 30.6.1808 (Str 8).
} 


\section{Die Konzeption der "gebildeten Stände"}

Was aber sollte mit dem (grund-)vermögenslosen Adel geschehen? Und wie konnte man den Ansprüchen eines nach vorn drängenden, leistungsfähigen Bildungsbürgertums gerecht werden, das sich im Vergleich zum alten Adel spätestens seit $1799 \mathrm{im}$ Staatsdienst auf allen Ebenen unterhalb der allerhöchsten Ränge schon mindestens ebenso viele, meist aber mehr „staatstragende“ Positionen gesichert hatte?

Auf beide Fragen gab es eine Antwort: Diese zweite Klasse des Adels mußte mit den sozialen Aufsteigern aus dem Bürgertum zu einer homogenen, mit gewissen, geringeren Privilegien ausgestatteten Schicht verschmelzen.

Schon die altbayerische Siegelmäßigkeit war seit langem kein spezifisches Adelsvorrecht mehr, sondern stand einem breiteren Kreis Privilegierter, wie bürgerlichen Räten, höheren Offizieren, Akademikern, Geistlichen und Patriziern zu ${ }^{\mathbf{4 0}}$. Hatte sich darüber hinaus die höhere, akademisch gebildete Beamtenschaft - ungefähr vom Kollegialrat an aufwärts - bereits bei der Neuorganisation des Beamtenrechts 1805/08 rechtlich und gehaltsmäßig von den unteren, nichtpragmatischen Staatsdienern deutlich abzugrenzen gewuß $\mathrm{t}^{\mathbf{4}}$, so hatten ihre Vertreter wenig später im allgemeinen nichts gegen die Fortexistenz oder gar die Wiederbelebung bestimmter Vorrechte, wie des Kadettenrechts, des privilegierten Gerichtsstandes oder der Selbstobsignation der Erbschaft eines Blutsverwandten ${ }^{42}$, einzuwenden - sofern nur derartige Rechte sich auch auf ihresgleichen erstreckten bzw. künftighin auf alle Angehörigen der „gebildeten Stände“, zu denen sie sich neben Stabsoffizieren und Adeligen natürlich ebenfalls rechneten, ausgedehnt wurden. Namentlich der nobilitierte Bauern- und Wirtssohn Zentner wirkte eifrig in diese Richtung und arbeitete beispielsweise den Entwurf jener Verordnung aus, die sämtlichen „Personen von einem ansehnlichen Stande“ in amtlichen Ausfertigungen das Prädikat „Herr" als Anrede zubilligte ${ }^{43}$. Um „für den gebildeten Unterthan eine andere Strafart als für den rohen Haufen einzuführen", wurde sogar eine neue Strafe, die Festungshaft, entwickelt ${ }^{44}$ ! Bei alledem zeigten sich jedoch auch die Angehörigen des „Feudaladels“ im Geheimen Rat als sehr entgegenkommend. Man darf also über die Konfrontation zwischen „Bürokratie“ und „Feudaladel“ keinesfalls die zunehmende Kooperation übersehen ${ }^{45}$.

${ }^{40}$ Codex juris Bavarici judiciarii de anno MDCCLIII, München ${ }^{2} 1771$, Kap. XX, $\S 9$, Nr. 7 ; Wiguläus Xaverius Aloysius Frbr. v. Kreittmayr, Anmerkungen über den Codicem Maximilianeum Bavaricum civilem, Tl. V, (München 1768) Kap. XXII, § 16.

${ }_{11}$ Dazu Demel, (wie Anm. 19) $109 \mathrm{ff}$.

12 Protokoll der Verfassungskommission v. 25.10.1814 (Str 1643); Protokoll der Zivilgesetzkommission v. 24.7.1814 (Str 2847).

${ }^{43}$ Vortrag Zentners v. 1.10.1812 (Str 289); VO v. 19.10.1812 (Rgbl. 1812, Sp. 1833-1835); vgl. dazu Bernd Wunder, Privilegierung und Disziplinierung. Die Entstehung des Berufsbeamtentums in Bayern und Württemberg (1780-1825), (Studien zur modernen Geschichte 21, München-Wien 1978) $160 \mathrm{ff}$.

11 So C. Graf Arco in der Sitzung der Geheimratssektionen des Innern und der Justiz v. 10.9. 1810 (Str 2359); Vgl. Wunder, (wie Anm. 43) 174 ff.

${ }^{13} \mathrm{Vgl}$. Eberbard Weis, Zur Entstehungsgeschichte der bayerischen Verfassung von 1818. Die Debatten in der Verfassungskommission von 1814/15, in: ZBLG 39 (1976) 413-444. 
Daß Montgelas und andere Regierungsmitglieder die Verschmelzung von Adel und Bildungsbürgertum nicht nur begrüßt, sondern sogar bewußt gefördert haben, steht außer Frage. Dies bezeugen nicht allein die über 200 Nobilitierungen der Ära Montgelas - charakteristischerweise großenteils in Form des Transmissionsadels -, sondern vielleicht noch mehr die Bemühungen des Ministers, reine Adelsinstitutionen wie bestimmte Damenstifte oder Ritterorden dem Zugang Bürgerlicher zu öffnen ${ }^{46}$, wobei etwa beim Münchner St.-Anna-Stift seit 1802 selbstverständlich wiederum primär die Töchter hoher bürgerlicher bzw. nobilitierter Beamter, später dann zeitweise ausschließlich Offizierstöchter in den Genuß der vielbegehrten neuen Präbenden kamen $^{47}$. Für die - so wörtlich - „weibliche Jugend der höheren Stände“ wurde in München sogar eine „Max-Joseph-Stift“ genannte eigene Erziehungsanstalt (neben einer anderen für die Töchter des „Bürgerstandes") gegründet ${ }^{48}$. Dieses staatlicherseits hochdotierte Institut, das gleichermaßen eben nur den "höheren Töchtern“ - also denjenigen des Adels, des Offizierskorps und der höheren Beamtenschaft vom Kollegialrat an aufwärts - vorbehalten war, unterstand übrigens direkt dem Innenminister und bekam später von König Ludwig I. gegenüber dem Universitätsgebäude eine neue repräsentative Heimstatt an der Ludwigstraße (heute Juristisches Seminargebāude).

Daß aber diese Anstalt als erste Leiterin eine Mme. Chardoillet erhielt, die zuvor in Ecouen an jener „Maison impériale“ unterrichtet hatte, die von Napoleon speziell für die weibliche Jugend der Mitglieder seiner Ehrenlegion geschaffen worden war, und daß das sozusagen „männliche Pendant" dazu, die 1805 gegründete Kadettenanstalt nomen est omen -, ein ebenso exklusives „bayerisches Saint-Cyr“ zur Ausbildung des Offiziersnachwuchses darstellte ${ }^{69}$, beweist nur einmal mehr die enge Verbindung $z$ wischen der französischen und der bayerischen Gesellschaftspolitik - auch wenn etwa die Rekrutierung der Nachwuchsoffiziere in Bayern schon seit längerem nach ähnlichen Herkunftskriterien erfolgt war ${ }^{50}$.

${ }^{46}$ Geheime Staatskonferenz v. 18.8.1808 (Str 8). Zu den Damenstiften: MA Damenstifte 17, 47a-51; Leo Günther, Der Übergang des Fürstbistums Würzburg an Bayern. Das Ende der alten und die Anfānge der neuen Regierung, (Würzburger Studien zur Geschichte des Mittelalters und der Neuzeit 2, Leipzig 1910) 88. Der St. Michaels-Orden erhielt ab 1808 sogar eine eigene Nebenklasse der Ehrenritter, die speziell für bürgerliche „Männer von Verdienst“ geschaffen wurde. Ludwig Trost, Die Geschichte des St. Michaels-Ordens in Bayern und der St. Michaels-Bruderschaft seit dem Jahre 1693 bis auf die Gegenwart, (Leipzig 1888) 71-73.

${ }^{4}$ Rgbl. 1802, Sp. 129-133; 1809, Sp. 729-731.

${ }^{68}$ Reskript v. 18.6.1809 (MInn 44703); zur Dotierung: MKu 20546 sowie die Staatsinventarisation und Statistik von 1818 (MF 13687). Joseph Heigenmooser, Uberblick der geschichtlichen Entwicklung des höheren Mädchenschulwesens in Bayern bis zur Gegenwart, (Berlin 1905) $40 \mathrm{ff}$; Berta Sachs, Pläne und Maßnahmen der Regierung des Königs Max I. Joseph im Mādchenschulwesen Altbayerns, (München-Leipzig 1914) $42 \mathrm{ff}$;; Max-Joseph-Stift München 150 Jahre, 1813-1963, o.O., o. J. (München 1963).

49 Marcel Dunan, Napoléon et l'Allemagne. Le système continental et les débuts du royaume de Bavière 1806-1810, (Paris ${ }^{2} 1948$ ) 90; vgl. Lorenz Kuchtner, Das königlich bayerische Kadettenkorps, o. O., o.J. (1956) bes. 16.

so Otto Bezzel, Das kurpfalzbayerische Heer von 1778-1803, (Geschichte des bayerischen Heeres 5, München 1930) $118 \mathrm{f}$. 
Den Einfluß, den derartige Erziehungseinrichtungen durch gleichartige Ausbildung, aber auch die Möglichkeit zur Anknüpfung vielleicht lebenslanger Freundschaften auf die Stabilität der bayerischen Oberschicht im 19.Jahrhundert gewinnen sollten, wird man ebenso wenig geringschätzen dürfen, wie etwa die verbindende Wirkung eines Vereins wie der 1802 gegründeten Münchner „Museumsgesellschaft“, die jahrzehntelang von Wittelsbacherprinzen über Minister, zahlreiche Staatsräte und viele andere hohe Beamte und Offiziere, mehrere mediatisierte Fürsten und ausländische Gesandte bis zu Domherren, bedeutenden Künstlern und führenden Mānnern der Wirtschaft praktisch alles umfaßte, was in der Hauptstadt Rang und Namen besaß ${ }^{51}$. Ähnlich „ständeübergreifend“ wirkte auch die wissenschaftliche Forschung - in Bayern nicht zuletzt getragen von der 1807 neu organisierten und dotierten bayerischen Akademie der Wissenschaften, der gerade damals zahlreiche Beamte angehör$\operatorname{ten}^{52}$.

Utberhaupt verband wohl nichts die „gebildeten Stände“ auf die Dauer mehr als gerade die Bildungspolitik, jedenfalls nachdem eben in diesem Jahr 1807 Zentner die Leitung des bayerischen Unterrichtswesens übernommen und der Neuhumanist Niethammer darauf einen wesentlichen Einfluß gewonnen hatte. Die Wende vom Philanthropismus zum Neuhumanismus im bayerischen Bildungswesen, konkreter: von einer stark auf die Realien hinorientierten einheitlichen Elementarbildung zur scharfen Trennung zwischen gutfundierten Anstalten für ,gelehrte Bildung“ und solchen, miserabel ausgestatteten, zur „Volkserziehung “53 mit dem daraus resultierenden „sozialen Numerus clausus" an den Universitäten ${ }^{34}$, entsprach zweifellos den Wünschen und Interessen der mehrheitlich am klassizistischen Bildungsideal hängenden „gebildeten Stände“. War nicht überhaupt der Klassizismus ein Ausdrucksmittel dieser neuen Oberschich ${ }^{35}$ - und damit die neuhumanistisch geprägte Bildungspolitik auch

${ }^{31}$ Vgl. die Mitgliederliste von 1814 (HerA 45). Näheres: Uwe Puscbner, Die Gesellschaft „Museum“ (1802-1947). Bemerkungen zu einer Münchner Lesegesellschaft in den ersten Jahrzehnten ihres Bestehens, in: Histor. Kommission des Börsenvereins (Hrsg.), Buchhandelsgeschichte 1982/2, (Börsenblatt für den Deutschen Buchhandel - Frankfurter Ausgabe - Nr. 55 v. 2.7.1982). Die ständeübergreifende Funktion z. B. von Lesegesellschaften, aber auch eines Geheimbundes wie des llluminatenordens, ist schon oft betont worden.

${ }_{52} \mathrm{Vgl}$. Hof- und Staatshandbuch des Königreichs Bayern 1819, (München o.J.) $522 \mathrm{ff}$.

${ }^{53}$ Georg Bögl, Der Wandel der Volksbildungsidee in den Volksschullehrplänen Bayerns. Von der Braun'schen Reform bis zur Mitte des 19.Jahrhunderts, (München 1929) bes. 77 ff., $129 \mathrm{ff.}, 158$; Josef Neukum, Schule und Politik. Politische Geschichte der bayerischen Volksschule 1818-1848, (München 1969) 23 ff.; Franz Dobmann, Georg Friedrich Freiherr von Zentner als bayerischer Staatsmann in den Jahren 1799-1821, (Münchner historische Studien, Abt. Bayerische Geschichte 6, Kallmünz 1962) bes. $63 \mathrm{ff}$.

54 Wunder, (wie Anm. 43) 199 ff. (zit. 199). Ein - keineswegs üppig lebender - Landshuter Student schätzte übrigens seinen finanziellen Jahresbedarf auf ca. 400 fl. (MF 19689).

${ }^{55}$ Vgl. Jutta Thinesse-Demel, Münchner Architektur zwischen Rokoko und Klassizismus. Untersuchung des Ubergangs vom Rokoko zu klassizistischem Formen- und Gedankengut unter besonderer Berücksichtigung des Hofoberbaudirektors Carl Albrecht von Lespilliez, (Miscellanea Bavarica Monacensia 90, München 1980) $47 \mathrm{ff} ., 189,194 \mathrm{f}$. Es ist wohl kaum ein Zufall, daß die Gründung der Akademie der schönen Künste ausgerechnet ebenfalls im Frühjahr 1808 erfolgte (vgl. Dunan, (wie Anm. 49) $98 \mathrm{f}$ ). 
ein Instrument, um sich vom „gemeinen Mann“, vom „Volk“, den „niederen Ständen“ bzw. dem „gemeinen Bürger- und Bauernstande“ abzusetzen?

Natürlich ist Bildung kein scharfes Kriterium zur sozialen Abgrenzung, und so war es etwa durchaus nicht unumstritten, inwieweit speziell Bankiers und Unternehmern der Zugang zu den Privilegien der Oberschicht offenstehen sollte. Aber daß trotz einer gewissen gesellschaftspolitischen Wende nach 1817, die z.B. aus dem starken Rückgang der Nobilitierungstätigkeit unter Ludwig I. sichtbar wird ${ }^{56}$, diese gerade durch die Politik der Montgelasära zusammengewachsene Führungsschicht schon relativ homogen geworden war, mögen die Worte I.v. Rudharts belegen, der 1825 schrieb, „im wesentlichen und im juridischen Sinne" wären in Bayern nur noch zwei Stände vorhanden, nāmlich ,1) der Adel, dem sich die Staatsdiener und Geistliche als Theile anschließen, und 2) die Gemeinen" ${ }^{\text {"57. }}$.

Die hier bei Rudhart anklingende enge Verflechtung der höheren, privilegierten Stände können folgende, sehr vorläufige statistische Feststellungen erhärten ${ }^{58}$ :

Fast die Hälfte aller 1820 immatrikulierten bayerischen Adelsfamilien besaß einen Adel, der nicht vor 1750 zurückreichte. Von den erfaßten 3177 erwachsenen mānnlichen Mitgliedern dieser Familien waren mindestens rd. 54,5\% aktive oder ehemalige Staatsdiener im zivilen oder militärischen Bereich (zumeist natürlich in Bayern selbst), $1,0 \%$ besaß $\dddot{m}$ ter am Hof, 4,0\% standen im kirchlichen Dienst bzw. hatten dort gestanden, 4,4\% hatten Stellungen in nunmehr mediatisierten Gebieten innegehabt oder dienten auch derzeit noch bei Standesherren bzw. Gutsherrschaften. Als bloße Rentenbezieher sind nur 9,3\% der hier behandelten Adeligen zu betrachten, von ihnen lebte ein kleinerer Teil zudem im Ausland. Bürgerlichen Unternehmerberufen gingen 4,1\% nach, ebenso viele anderen bürgerlichen Beschäftigungen, z. B. als Advokaten, Arrte etc. 4,8\% standen in Ausbildung oder waren sonst noch unversorgt, von der gleichen Anzahl liegen überhaupt keine, von 8,9\% nur unklare Berufsangaben vor. Von letzteren stand jedoch sicherlich ebenfalls ein Teil im Staatsdienst, der damit ganz offenkundig eine überragende Bedeutung für den gesamten Stand besaß.

Gerade ein Großteil der Militärs war übrigens unverheiratet, insgesamt dürften immerhin noch gut $42 \%$ aller erwachsenen männlichen Adeligen ledig gewesen sein. Die rd. $45 \%$ Verheirateten hatten zu grob einem Viertel die Ehe mit einer Bürgerlichen geschlossen, doch zeigen sich hier in der Adelshierarchie charakteristische Un-

${ }^{56}$ Vgl. die Statistik HerA 126. Allerdings dachte auch Ludwig I. an eine Adelsreform nach englischem Vorbild. Er scheiterte aber am Widerstand einer konservativen Adelspartei in der ersten Kammer. Hubert Ostadal, Die Kammer der Reichsräte in Bayern von 1819 bis 1848. Ein Beitrag zur Geschichte des Frühparlamentarismus, (Miscellanea Bavarica Monacensia 12, München 1968) 9f.; Leonbard Lenk, Das Modell England in der bayerischen Verfassungsdiskussion zwischen 1770 und 1818, in: Richard van Dülmen (Hrsg.), Gesellschaft und Herrschaft, (Festgabe für Karl Bosl zum 60. Geburtstag, München 1968) 271-299, hier 289. Vgl. auch: Carl August Graf v. Drechsel, Ueber Entwürfe zur Reorganisation des deutschen Adels im 19. Jahrhundert, (Ingolstadt 1912) bes. $16 \mathrm{ff}$.

${ }^{57}$ Ignaz v. Rudbart, Ueber den Zustand des Königreichs Baiern nach amtlichen Quellen, 3 Bde., (Stuttgart-Tübingen 1825/27) hier Bd. I, 46.

${ }^{38}$ Nach der bayerischen Adelsmatrikel, (s. Anm. 8). 
terschiede: kein Fürst, nur ca. 3\% der Grafen und 9\% der Freiherren hatten als Adelige bürgerlich geheiratet, jedoch 36-43\% aller untitulierten Adeligen - sogar ohne die nur persönlich oder auf Transmission Nobilitierten! Mit anderen Worten: zumindest in den unteren Adelsklassen dürfte es kaum Adelige gegeben haben, die nicht bürgerliche Verwandte bzw. Verschwägerte besaßen.

Daß jedoch an die Stelle der alten Exklusivität wenigstens teilweise eine neue getreten war, darf man nach der Feststellung vermuten, daß von 115 bürgerlich verheirateten Adelstöchtern über $62 \%$ einen Mann geheiratet hatten, der eine höhere Ausbildung besaß, weitere $16,5 \%$ einen nichtakademischen (in der Regel mittleren) Beamten - auch viele Adelige selbst standen ja nur auf diesem Dienstrangniveau. Jedenfalls waren demnach knapp $2 / 3$ innerhalb eines Personenkreises verheiratet, der unzweifelhaft den „gebildeten Ständen“ zuzurechnen ist, dazu kommen noch 13\% Heiraten mit bürgerlichen Unternehmern, die zumindest bedingt dieser Schicht zugezählt werden können. Dieses Heiratsverhalten weist also ganz in dieselbe Richtung, in die auch die Adelspolitik Montgelas' ging.

\section{Ausblick}

Diese Adelspolitik hatte, wie gesagt, teilweise unter dem Druck Napoleons Gestalt angenommen, und sie besaß dementsprechend zweifellos auch das Ziel, die neue Elite des Rheinbundstaates Bayern mit derjenigen des Empire zu verzahnen. Bildeten nicht die bayerischen Verdienstordensträger ebenfalls eine Art Ehrenlegion mit Ehrenpensionen etc., und waren nicht zahlreiche bayerische Politiker und Militärs in die französische Ehrenlegion aufgenommen worden (samt Pensionsbezug!), wie auch umgekehrt viele Franzosen einen bayerischen Verdienstorden erhielten - 148 allein in den Jahren 1806/13 den Militär-Max-Joseph-Orden, das militärische Gegenstück zum Zivilverdienstorden 39 ?

Diese engen Verflechtungen wurden freilich ab 1813 aufgelöst, die neue Ordnung im Adelswesen beider Staaten aber blieb im Prinzip erhalten. Denn hatte nicht auch Napoleon sich bemüht, aus Elementen des alten Adels und des aufstrebenden Bürgertums - speziell auch des Bildungsbürgertums - für sein Land eine neue Elite zu formen $^{60}$ ? War sein Gesellschaftsmodell nicht jenem von Montgelas konzipierten ähnlich gewesen, das sich vielleicht folgendermaßen veranschaulichen läßt?

39 Baptist Scbrettinger, Der Königlich Bayerische Militär-Max-Joseph-Orden und seine Mitglieder, (München 1882) 55 ff.; vgl. Dunan, (wie Anm. 49) 90, 470, Anm. 45; Bekanntmachung v. 18.8.1805 (Rgbl. 1805, Sp. 850-855, bes. Sp. 853); zu den Dotationen: MF 19712.

${ }^{60} \mathrm{Vgl}$. schon Dunan, (wie Anm. 49) 88. Zur gezielten Elitebildung in Frankreich: Durye, Godechot, (beide wie Anm. 26); Werner Giesselmann, Die brumairianische Elite. Kontinuität und Wandel der französischen Führungsschicht zwischen Ancien Régime und Julimonarchie, (Industrielle Welt 18, Stuttgart 1977) bes. 42 ff., 51, 501; Rolf Reichardt, Zu einer Sozialgeschichte der französischen Aufklärung, in: Francia 5 (1977) 231-249. 


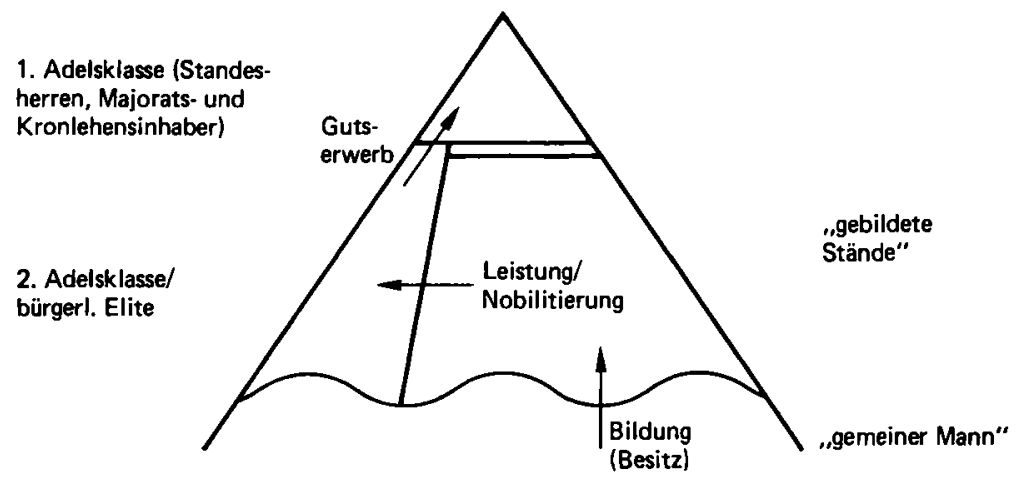

Nicht zuletzt dieses, dynamische und statische Elemente verbindende Gesellschaftskonzept scheint mir die relative soziale Stabilität Bayerns im 19.Jahrhundert gewährleistet zu haben. Damit aber hätte Montgelas in der Tat das Ziel erreicht, das er sich mit seiner Gesellschaftspolitik gesetzt hatte, nāmlich „l'équilibre qui s'est déjà rétabli et se rétablira encore mieux dans l'ordre social ${ }^{\text {“61 }}$.

${ }^{61}$ Laubmann, Doeberl, (wie Anm. 6) 130. 